and satellite based services are extremely popular. The astronomy community will also have to get more involved in the various regulatory fora, and establish good contacts with industry. To do so will require that more human as well as financial resources be dedicated to spectrum management activities.

\title{
8. JAPANESE PERSPECTIVE FOR INTERFERENCE TO RADIO ASTRONOMY
}

\section{K. Kawaguchi (NRAO, National Astronomical Observatory)}

Interference to radio astronomical observations becomes serious, especially in relatively low frequency regions. Since general discussions on interference are given in other papers of this conference, the present paper describes some examples of interference detected in recent astronomical observations and future perspective.

I. Spurious in fringe detection between Kashima and VSOP in $1600 \mathrm{MHz}$ region Recently, ISAS (Institute of Space and Aeronautics) launched a spacecraft HALCA with 8-m radio telescope for very long baseline interferometer. The first fringe test was carried out on 1997 May 7 for VSOP (VLBI Space Observatory Program) using the Usuda 64- $\mathrm{m}$ and Kashima 34-m ground radio telescopes and HALCA space telescope. In the test, the Kashima telescope received extended spurious noises at $1642.5 \mathrm{MHz}$, which made it difficult to detect fringes between HALCA and Kashima. Then the observation frequency was shifted to $1670-1686 \mathrm{MHz}$ to avoid spurious in Kashima, and fringes were successfully detected between Kashima and HALCA. The band is protected as radio-astronomical band, but it is noted that such strong spurious are present for interference the astronomical observations.

II. Spurious in Taurus Molecular Cloud-1 observations with Nobeyama 45-m telescope The telescope site Nobeyama was chosen by considering to be free from radio interference. The site is surrounded with mountains and sparsely populated. However, it is found that recent development of various communication systems give large interference to radio observations below $30 \mathrm{GHz}$ region. Especially when we observe quiet, cold dark astronomical source like TMC-1, where spectral line width are narrow as $0.6 \mathrm{~km} \mathrm{sec}{ }^{-1}(60 \mathrm{kHz}$ at $30 \mathrm{GHz})$, it becomes very difficult to discriminate real lines from artificial spurious emission, even if a position switching method is employed for astronomical objects.

III. 95-GHz cloud profiling radar CRL (Communication Research Laboratory, Japan) and NASDA (National Space Development Agency of Japan) have project of a cloud profiling radar system from satellite for investigation of global warming which can be emotive issues. The radar system will use $3 \mathrm{~mm}$ radiation by considering scattering effect and established technology levels.

The frequency is also very important for radio astronomical observations. The following interference will occur,

(1) Main lobe-main lobe coupling

In the case of the Nobeyama 45-m telescope, the received power from the radar is estimated to be about $1 \mathrm{~W}$ by considering the sizes of radar beam and telescope. Since the destruction power for SIS (Superconductor-Insulator-Superconductor) junction is estimated about $10 \mathrm{~mW}$, the radar power is two order of magnitude larger than the destruction power.

(2) Main lobe (telescope)-side lobe (radar) coupling

This is the case that a telescope sees a radar side lobe. Usually an SIS mixer receiver is working with a local power of $10^{-9} \mathrm{~W}$, and higher local power gives a change in bias point and results in increase of noise temperature.

(3) Side lobe-side lobe coupling

This is the case that a telescope receives a power less than receiver saturation level, but the radar radiation is observed as a spurious line at the exact radar frequency. The telescope receives more power than harmful interference level $(\mathrm{Pr}=-203 \mathrm{dBW}$ at $90 \mathrm{GHz}$ region), for a shorter distance than $1080 \mathrm{~km}$. If the satellite radar system wants to use the $95 \mathrm{GHz}$ band, the band must be allocated to active sensing from satellite. From astronomical points of view, we are worry about such thing that many active services use the $95 \mathrm{GHz}$ region after the allocation. The $95 \mathrm{GHz}$ region is also not allocated for radioastronomical bands, but the effects (1) and (2) from radar make it difficult to observe in radio astronomical bands of $3 \mathrm{~mm}$ region. We must make efforts to reallocate the $95 \mathrm{GHz}$ band for astronomical bands, because of protection of the best observing bands. Alternative way is to reserve the millimeter-wave telescope sites as protection zones. 